\title{
Dusty LBV Nebulae: Tracing the Mass Loss History of the Most Massive Stars
}

Laurens B.F.M. Waters ${ }^{1,2}$, Robert H.M. Voors ${ }^{3}$, Patrick W. Morris ${ }^{3,1}$, Norman R. Trams ${ }^{4}$, Alex de Koter ${ }^{1}$ and Henny J.G.L.M. Lamers ${ }^{3}$

1 Astronomical Institute 'Anton Pannekoek', University of Amsterdam, Kruislaan 403, NL-1098 SJ Amsterdam, The Netherlands

2 SRON Laboratory for Space Research Groningen, P.O. Box 800, NL-9700 AV Grogingen, the Netherlands

3 SRON Laboratory for Space Research Utrecht, Sorbonnelaan 2, NL-3584 CA Utrecht, the Netherlands

4 Astrophysics Division, ESA Space Science Department, ESTEC, Keplerlaan 1, Noordwijk, The Netherlands

\begin{abstract}
We present spectra obtained with the Infared Space Observatory (ISO) of the dust shells surrounding several Luminous Blue Variables (LBVs), both in our galaxy and in the LMC. The 20-45 $\mu \mathrm{m}$ spectra of R71, AG Car and Wra 751 show prominent emission features from crystalline silicates. The composition of the crystalline silicates in LBV dust shells is compared to that found in other types of objects, such as (post)-AGB stars and red supergiants (RSG). Both Wra 751 and AG Car have a high ratio of pyroxenes to olivines. This suggests that the grains in both stars experienced very similar processing, but that this processing has lead to a higher abundance of pyroxenes compared to RSG. The dust composition of the three LBVs discussed here suggests dust formation in a cool outflow not unlike those of RSG.
\end{abstract}

\section{Introduction}

Luminous Blue Variables (LBVs) are massive $\left(\mathrm{M}_{\mathrm{zams}}>50 \mathrm{M}_{\odot}\right)$, hot supergiants with dense, radiation-driven ionized winds. They show remarkable variations in temperature and radius, at roughly constant luminosity, with timescales of several years. LBVs are believed to be a short-lived post-mainsequence evolutionary phase of the most massive stars in galaxies; they will probably evolve to the He-rich Wolf-Rayet (WR) phase. There are on the order of $10 \mathrm{LBVs}$ and LBV candidates known in our galaxy (Humphreys \& Davidson 1994); well studied cases are P Cygni, $\eta$ Car, and AG Car. For recent reviews on LBVs see Luminous Blue Variables: massive stars in transition, eds. A. Nota \& H.J.G.L.M. Lamers (1997).

Almost all LBVs are associated with dusty ring nebulae (Nota et al. 1995). These nebulae are the result of previous phases of very high mass loss and/or of LBV outbursts, such as that of $\eta$ Car around 1840. Thus, the shape, kinematics and composition of LBV ring nebulae can be used to trace their integrated mass loss history. Recently, the origin of LBV nebulae has received 
increased attention because the nebular gas-phase abundances of $\mathrm{N}$ and $\mathrm{O}$ in some nebulae are not in agreement with evolutionary predictions (Smith 1997; Smith et al. 1998). The mild N enhancements found in e.g. AG Car are better explained by a short Red Supergiant (RSG) phase during which the star was fully convective. Independently, Waters et al. $(1997 ; 1998$ a) have shown that the dust in several LBV ring nebulae (among which AG Car) contains small amounts of crystalline silicates; these grains are also seen in the winds of RSG and AGB stars, suggesting similar physical and chemical conditions in the dust forming layers.

This paper discusses infrared spectroscopy taken with the Short Wavelength Spectometer (SWS, de Graauw et al. 1996) and with ISOCAM (Cesarsky et al. 1996) on board of the Infrared Space Observatory (ISO, Kessler et al. 1996) of two galactic LBVs (AG Car and Wra 751) and one LMC LBV (R71). These spectra yield new information about the composition of the dust through their solid state emission features. We compare the LBV dust spectra to those of NML Cyg (a RSG) and IRC+10 420 (a post-RSG).

\section{Infrared spectroscopy of LBVs}

\subsection{AG Car and Wra 751}

AG Car and Wra 751 are among the best studied galactic LBVs. Both stars have a prominent bipolar ring nebula expanding at $65-70 \mathrm{~km} / \mathrm{s}$ (AG Car; Nota et al. 1992) and about $25 \mathrm{~km} / \mathrm{s}$ (Wra 751; Hutsémekers \& van Drom 1991; Garcia-Lario et al. 1998), and have strong infrared excess due to thermal emission from dust grains in their shell (e.g. McGregor et al. 1988; Hu et al. 1990). The LBV nature of Wra 751 is still somewhat uncertain, since it does not show the characteristic variations in temperature (Garcia-Lario et al. 1998). ISO-SWS spectra of both objects were discussed previously by Lamers et al. (1996) and Waters et al. $(1997,1998)$; these observations show the presence of weak, narrow emission bumps in the 30-45 $\mu \mathrm{m}$ region due to crystalline silicates. In the case of Wra 751, also the $9.7 \mu \mathrm{m}$ amorphous silicate feature is present. In figure 1, we show new ISO-SWS observations of AG Car, as well as a ground-based $12.5 \mu \mathrm{m}$ narrow-band $\mathrm{N} 3$ image taken with TIMMI (Käufl et al. 1992) at the $3.6 \mathrm{~m}$ telescope, ESO, La Silla. The SWS spectrum is better centered on the nebula than the spectrum presented previously.

The better quality data for AG Car allows us to re-determine the shape and strength of the emission bands in the 30-38 $\mu \mathrm{m}$ window region. In figure 2 , we show continuum subtracted 30-38 $\mu \mathrm{m}$ spectrum of AG Car. The presence of relatively narrow emission bands near 33 , and $35 \mu \mathrm{m}$ is evident. Note that the $33.8 \mu \mathrm{m}$ band reported by Waters et al. (1997) is not seen in the speed 4 spectrum, nor in the (re-reduced) speed 1 spectrum (not shown). The differences between the present paper and previous results is due to improved data reduction and continuum subtraction procedures. We have 

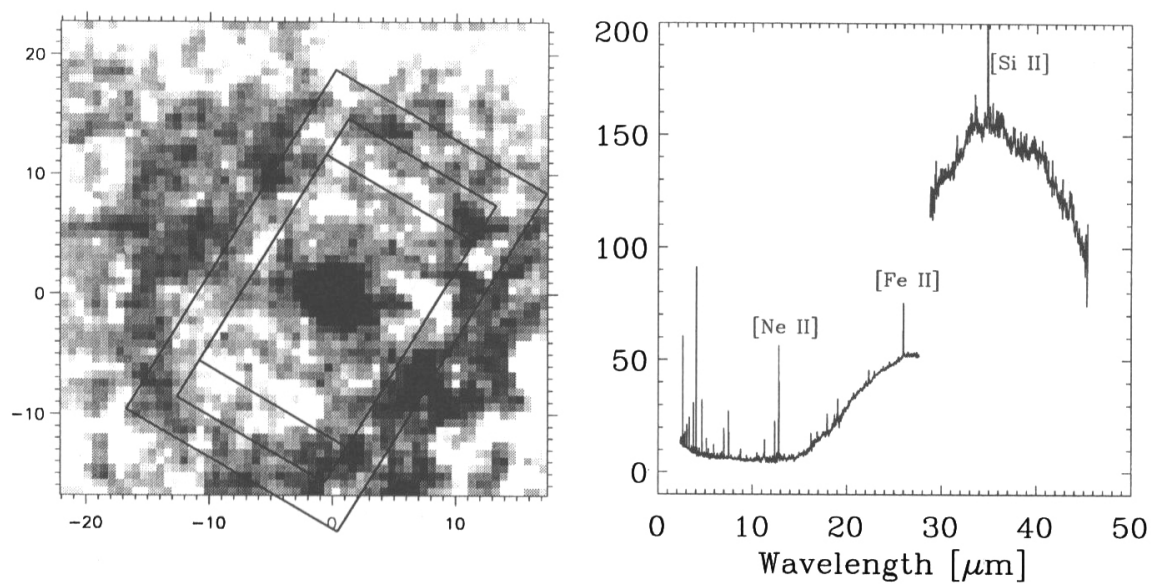

Fig. 1. left panel: TIMMI $12.5 \mu \mathrm{m}$ image of AG Car. The scale is in arcsec. Overplotted is the position and size of the ISO-SWS apertures. Note the bi-polar structure of the nebula. right panel: ISO-SWS AOT01 speed 4 spectrum of AG Car. The jump at $30 \mu \mathrm{m}$ is due to a change to a larger aperture of the SWS. Flux scale is in Jy.

compared the emission bands to laboratory spectra of crystalline silicates, and find a good match with the pyroxene ortho-enstatite $\left(\mathrm{MgSiO}_{3}\right)$ spectrum published by Koike and Shibai (1998). The laboratory results of Jäger et al. (1998) for a slightly Fe-enriched ortho-enstatite also give a reasonable match to the wavelengths of the bands, but the band strengths of these laboratory data do not agree with the AG Car data. It is remarkable that the $33.8 \mu \mathrm{m}$ crystalline olivine forsterite $\left(\mathrm{Mg}_{2} \mathrm{SiO}_{4}\right)$ peak, which is prominent in almost all oxygen-rich dust shells with crystalline silicates (see e.g. Waters et al. 1996), is weak or absent in AG Car. This suggests that the abundance ratio olivines to pyroxenes is much lower in AG Car than in other sources. We will return to this point below.

The ISO-SWS spectrum of Wra 751, and ground-based $10 \mu \mathrm{m}$ imaging using TIMMI, have already been presented by Waters et al. (1998). The continuum subtracted spectrum is shown in figure 2. The pattern of emission features resembles that of AG Car, and we can draw similar conclusions concerning the lack of forsterite (see also Waters et al. 1998). Also the width of the emission bands is comparable in both objects. The similarity in solid state emission features in both stars implies that the formation and thermal 


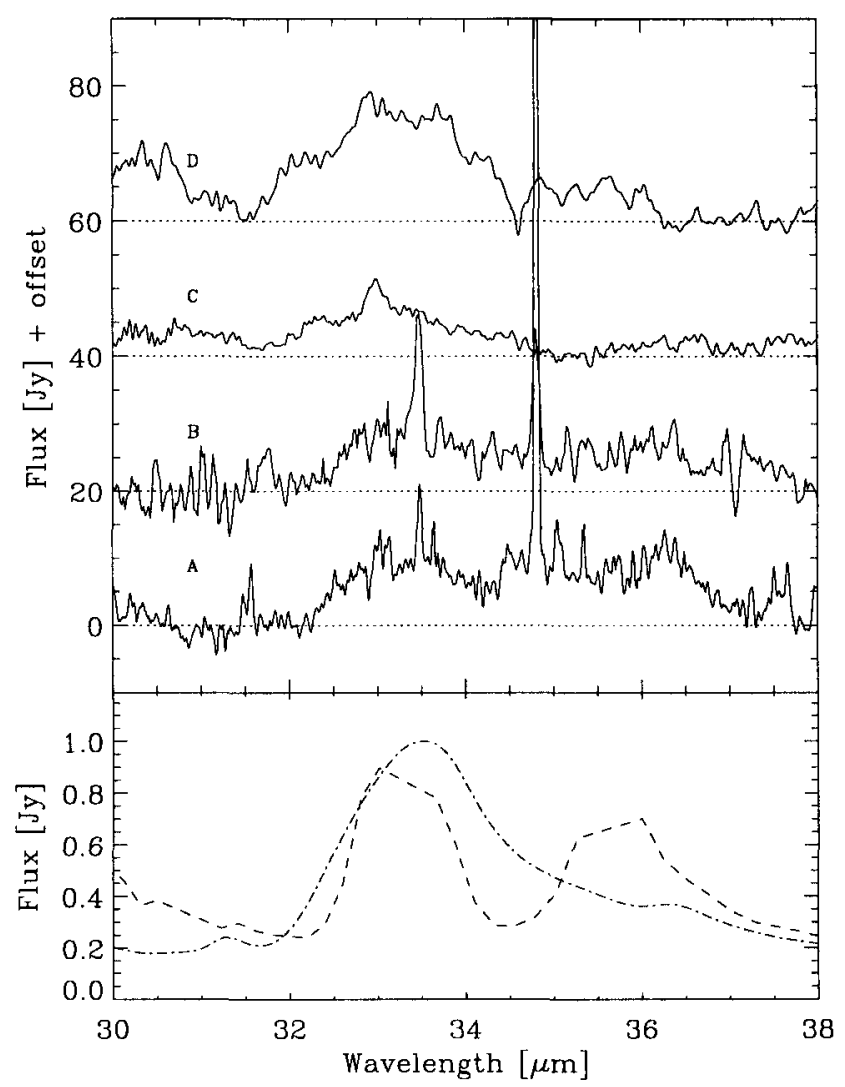

Fig. 2. Top: continuum subtracted 30-38 $\mu \mathrm{m}$ spectra of AG Car (slow scan) (A), Wra 751 (B), IRC+10 420 (C), and NML Cyg (D). Also shown are laboratory measurements of forsterite (dash-dotted line; Jäger et al. 1998) and enstatite (dashed line; Koike \& Shibai 1998). The LBVs have little or no forsterite

processing history of the crystalline silicate grains is similar for both stars. AG Car and Wra 751 share other properties: the shape and kinematics of the nebulae, and the evolutionary phase in which both stars presently are. Although we are dealing with only two objects, these similarities are encouraging since it strengthens the diagnostic value of the solid state emission bands. 


\section{$2.2 \quad$ R 71}

The LMC LBV R71 is one of the brightest stellar sources in the LMC at 25 $\mu \mathrm{m}$ (Wolf \& Zickgraf 1986), due to the presence of a warm, detached dust shell with a dust mass of about $510^{-3} \mathrm{M}_{\odot}$ (Hutsemékers 1997), corresponding to a total mass of about $1 \mathrm{M}_{\odot}$ (depending on the uncertain gas/dust ratio). Roche et al. (1993) detected $10 \mu \mathrm{m}$ silicate emission and conclude that the dust is oxygen-rich. We have observed R7I with ISO-SWS and with ISOCAM and will report on these observations elsewhere (Waters et al. in preparation). Here we mention the detection of a prominent $23 \mu \mathrm{m}$ feature due to crystalline silicates. To our knowledge this is the first extragalactic detection of this band. We have fitted the dust spectrum using a 1D radiative transfer code, and assuming $\mathrm{T}_{\text {eff }}=17,000 \mathrm{~K}$ and $\mathrm{L}=710^{5} \mathrm{~L}_{\odot}$ (Lennon et al. 1994). We find a best-fit model with only large grains $(0.1$ to $1 \mu \mathrm{m})$ to fit the amorphous silicate feature, and derive a dust mass of $210^{-2} \mathrm{M}_{\odot}$, resulting in a total mass of $2 \mathrm{M}_{\odot}$ (for a gas/dust ratio of 100 , which is very uncertain). The time-averaged total mass loss rate is $710^{-4} \mathrm{M}_{\odot} / \mathrm{yr}$ for a period of about $310^{3}$ years and stopped 6000 years ago. We have neglected the effects of the interaction between the present-day fast wind and the slowly expanding dust shell in these calculations. Also, the outer radius of the dust shell is poorly constrained, which affects the estimate of the duration of the mass loss and the mass loss rate. However, the characteristics of the dust shell agree well with those expected for a RSG wind. Therefore R71 could have been a RSG when it produced the dust shell.

The ISOCAM data reveal the presence of a warm dust component which cannot be fitted with our radiative equilibrium dust model. This points to the presence of a dust component close to the star, or to a separate population of small grains out of thermal equilibrium.

\section{PAH emission in LBVs}

To our surprise, the new high quality SWS spectrum of AG Car shows clear evidence for the presence of the well-known family of emission bands near 3.3, 6.2, 7.7, 8.6 and $11.3 \mu \mathrm{m}$. The 5-12 $\mu$ CAM CVF spectrum of R71 also shows these bands (see figure 3 ). These bands are usually attributed to Polycyclic Aromatic Hydrocarbons (Leger \& Puget 1984). PAH emission was previously found in the nebula of the candidate LBV HD168625 (Skinner 1997). The warm dust continuum present in both stars near $10 \mu \mathrm{m}$ is probably related to a population of small grains as well.

The band strengths of the PAHs can be used to derive constraints on the nature of the grains and on their excitation (e.g. Allamandola et al. 1989). AG Car has a rather prominent $11.3 \mu \mathrm{m} \mathrm{C-H}$ out-of-plane bending mode peak compared to the strength of e.g. the $7.7 \mu \mathrm{m} \mathrm{C-C} \mathrm{stretch} \mathrm{band} \mathrm{strength;}$ such a strong band can be due to e.g. a population of very large PAHs, or to predominantly neutral PAHs. The ratio of the $3.3 \mu \mathrm{m} \mathrm{C}-\mathrm{H}$ stretch to 11.3 
$\mu \mathrm{m}$ band strength is between 3 and 4 , which rules out the presence of large PAHs, and suggests that the PAHs are neutral.

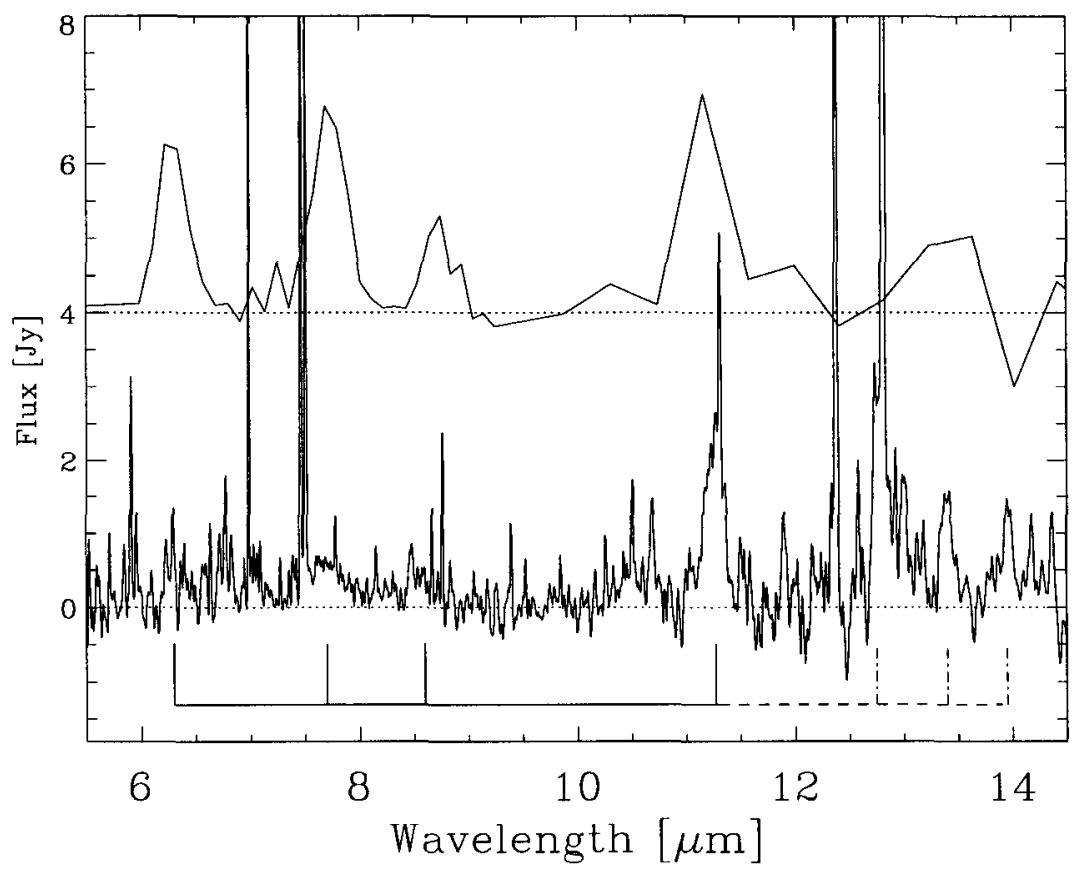

Fig. 3. PAH emission bands in R 71 (top) and AG Car (bottom). For R 71 we multiplied the spectrum by a factor 50 and we removed the prominent amorphous silicate band for clarity. Tickmarks indicate the expected position of the bands

In $\mathrm{R} 71$, the $11.3 \mu \mathrm{m}$ band is strong but our data do not cover the 3.3 $\mu \mathrm{m}$ region; In addition, the $11.3 \mu \mathrm{m}$ PAH band may blend with the 11.3 $\mu \mathrm{m}$ forsterite band. Given the strength of the $23 \mu \mathrm{m}$ forsterite band and the relatively high temperature of the dust, the flux in this feature could be significant. Therefore, we cannot conclude much about the size of the PAHs in R71.

The presence of PAH emission in circumstellar envelopes is strongly correlated with C-rich chemistry: PAHs are abundant in C-rich planetary nebulae (PNe) and post-Asymptotic Giant Branch (AGB) stars. It is therefore not obvious that such C-bearing grains are present in the O-rich environment of the LBV nebulae. It is interesting to note that ISO spectroscopy of evolved C-rich objects with prominent PAH emission has shown that this 'mixed chemistry' is more widespread than previously thought: several [WC] central stars of PNe as well as the Red Rectangle nebula show a combination 
of oxygen-rich crystalline silicates and strong PAH emission (Waters et al. 1998b, 1998c; Cohen et al. 1999). In low mass stars, this can be understood in terms of the chemical evolution of the AGB star, which towards the end of the AGB changes from O-rich to C-rich chemistry. The LBVs discussed here are not yet C-rich. However, evolutionary calculations (Meynet et al. 1994) indicate that for an $\mathrm{M}_{\mathrm{ZAMS}} 85 \mathrm{M}_{\odot} \mathrm{LBV}$ the photospheric $\mathrm{C} / \mathrm{O}$ ratio is about 0.8 , i.e. above solar. Perhaps the grains are produced in the shock that occurs when the present-day C-enhanced fast wind collides with the slower dusty ejecta. This shock could drive a non-equilibrium C-rich chemistry which would result in the production of PAH molecules. Unfortunately, we have no information about the spatial distribution of the PAHs in the nebulae to verify this hypothesis.

In several galactic RSG weak PAH emission at $11.3 \mu \mathrm{m}$ has been reported (Sylvester et al. 1994), proving that these molecules can be produced in an O-rich environment.

\section{Comparison with other evolved dusty envelopes}

In figure 2 we compare the $30-38 \mu \mathrm{m}$ solid state emission bands of Wra 751 and AG Car to those of the M supergiant NML Cyg and the peculiar A-F type supergiant IRC+10 420. The latter object is believed to be a transition object, rapidly evolving to the blue part of the HR diagram (e.g. Jones et al. 1993). It is very well possible that $I R C+10420$ is a WR progenitor. Both NML Cyg and IRC+10 420 have or have had mass loss rates in excess of $10^{-4}$ $\mathrm{M}_{\odot} / \mathrm{yr}$ (Justtanont et al. 1996; Oudmaijer et al. 1996). It is evident that all four sources have crystalline silicates, but the ratio of the 33.0 (pyroxene) to $33.8 \mu \mathrm{m}$ (olivine) peaks is different: in NML Cyg the olivine and pyroxene peaks are similar in peak strength, while in IRC +10420 the olivine peak is quite weak. The olivine peaks seen in AGB and post-AGB stars (Waters et al. 1996) are significantly more prominent. Indeed, RSG and AGB stars have more olivines than Wra 751 and AG Car (Molster et al., in preparation).

In order to understand the differences in composition of the crystalline silicates between Wra 751 and AG Car on the one hand, and RSG and AGB stars on the other hand, it is useful to consider the physical and chemical conditions in the dust forming layers of dusty outflows. Crystalline silicates form predominantly in winds with very high mass loss rates, i.e. high densities in the dust forming layers (the LBVs are no exception to this trend). In order for the crystal lattice to become ordered after condensation from the gas phase, the temperature of formation must be higher than the glass temperature, which is about $1050 \mathrm{~K}$ for silicates. Therefore the crystalline grains must be among the first to condense from the gas phase. An important point to note is that crystalline silicates are $\mathrm{Mg}$-rich and Fe-poor, while amorphous grains are Fe-rich and $\mathrm{Mg}$-rich. A possible scenario for the dust condensation could be that first crystalline grains condense, and that most, but not all, of these 
grains absorb Fe at temperatures of about $1000 \mathrm{~K}$ or slightly lower, which destroys the ordered lattice structure (Tielens et al. 1998). The reaction with $\mathrm{Fe}$ is not possible for higher temperatures.

The condensation temperature for the $\mathrm{Mg}$-rich olivine forsterite is about $1500 \mathrm{~K}$. Slow cooling of this material results in the formation of the Mg-rich pyroxene enstatite. Therefore, the thermal processing timescale of crystalline grains after formation may determine the abundance ratio between olivines and pyroxenes. For the lower mass AGB stars and for RSG, the higher abundance of olivines may therefore indicate a more rapid cooling of the grains after formation compared to the LBVs Wra 751 and AG Car. This may be related to differences in stellar temperature, to the presence or absence of a chromosphere, and to the optical depth of the dust shell.

Perhaps the most surprising conclusion to be drawn from the ISO spectra is the similarity in physical and chemical conditions that must have occurred during the formation of the dust in RSG and LBVs. A picture emerges of a slow outflow at high density around a cool star. Simple models for the dust shell surrounding R71, AG Car and Wra 751 (Waters et al. 1997, 1998a), suggest that these shells could have been produced during a brief $\left(210^{3} \mathrm{yrs}\right.$ for AG Car) phase of very high mass loss. A more difficult question to answer is at what $T_{\text {eff }}$ the star produced the dust shell. The high abundance of pyroxenes may point to an on average higher $T_{\text {eff }}$ for the LBVs compared to RSG, but this is by no means certain. A possible scenario for the production of LBV ring nebulae could therefore be a brief period as yellow or red surpergiant with a very high mass loss rate. Observationally, such objects would be very rare. For several LBVs it has now been established that the grain size is large $(1 \mu \mathrm{m})$. Such grains produce grey extinction at optical wavelengths, i.e. the star would not appear to be very reddened. Perhaps the signature of LBVs during the ejection of the ring nebula is a yellow supergiant with modest circumstellar reddening but significant extinction, surrounded by a warm dust shell. This would not be in conflict with the observed lack of very luminous red supergiants in galaxies (Humphreys \& Davidson 1979).

Acknowledgements. The authors thank Xander Tielens, Frank Molster, Teije de Jong, Jeroen Bouwman and Douwe Beintema for their help. TdJ kindly made the NML Cyg data available to us. LBFMW and AdK acknowledge financial support from an NWO Pionier grant.

\section{References}

Allamandola, L.J., Tielens, A.G.G.M., Barker, J.R.: 1989, ApJS 71, 733

Cesarsky, C.J. et al.: 1996, A\&A 315, L32

Cohen, M., Barlow, M., Sylvester, R.J., Liu, X.-W., Cox, P., Schmitt, B., Speck, A.K.: 1999, ApJ (submitted)

De Graauw, Th. et al.: 1996, A\&A 315, L49

Garcia-Lario, P., Riera, A., Manchado, A.: 1998, A\&A 334, 1007 
Hu, J.Y., De Winter, D., Thé, P.S., Pérez, M.R.: 1990, A\&A 227, L17

Humphreys, R.M., Davidson, K.: 1979, ApJ 232, 409

Hutsemékers, D., van Drom, E.: 1991, A\&A 251, 620

Hutsemékers, D.: 1997, in Luminous Blue Variables: Massive Stars in Transition, eds. A. Nota \& H.J.G.L.M. Lamers, ASP Conference series vol. 120, p . 316

Jäger, C., Molster, C., Dorschner, J., Henning, Th., Mutschke, H., Waters, L.B.F.M.: 1998, A\&A, in press

Jones, T.J., et al.: 1993, ApJ 411, 323

Justtanont, K., et al.: 1996, A\&A 315, L217

Käuf, H-.U., Jouan, R., Lagage, P.O., et al., 1992, ESO messenger 70, 67

Kessler, M.F., et al., 1996 A\&A 315, L27

Koike, C., Shibai, H.,: 1998, in press

Lamers, H.J.G.L.M. et al.: 1996a, A\&A 315, L225

Leger, A., Puget, J.L.: 1984, A\&A 137, L5

Meynet, G., Maeder, A., Schaller, G., Schaerer, D., Charbonnel, C.: 1994, A\&AS 103,97

McGregor, P.J., Finlayson, K., Hyland, A.R., Joy, M., Harvey, P.M., Lester, D.F.: 1988, ApJ 329, 874

Nota, A., Leitherer, C., Clampin, M., Greenfield, P., Golimowski, D.A.: 1992, ApJ 398,621

Lennon, D.J., Wobig, D., Kudritzki, R.-P., Stahl, O., 1994, Space Sc. Rev. 66, 207

Nota, A., Livio, M., Clampin, M., Schulte-Ladbeck, R.: 1995, ApJ 448, 788

Oudmaijer, R.D., Groenewegen, M.A.T., Matthews, H.E., Blommaert, J.A.D.L., Sahu, K.C.: 1996, MNRAS 280, 1062

Roche, P.,F., Aitken, D.K., Smith, C.H.: 1993, MNRAS 262, 301

Skinner, C.J.: 1997, in Luminous Blue Variables: Massive Stars in Transition, eds. A. Nota \& H.J.G.L.M. Lamers, ASP Conference series vol. 120, p . 322

Smith, L.J.: 1997, in Luminous Blue Variables: Massive Stars in Transition, eds. A. Nota \& H.J.G.L.M. Lamers, ASP Conference series vol. 120, p . 311

Smith, L.J., Stroud, M.P., Esteban, C., Vilchez, J.M.: 1998, MNRAS 290, 265

Sylvester, R.J., Barlow, M.J., Skinner, C.J.: 1994, MNRAS 266, 640

Tielens, A.G.G.M., Waters, L.B.F.M., Molster, F.J., Justtanont, K.: 1998, Astrophys. Sp. Sc. 255,415

Waters, L.B.F.M., et al.: 1996, A\&A 315, L361

Waters, L.B.F.M., Morris, P.W., Voors, R.H.M., Lamers, H.J.G.L.M.: 1997, in $\mathrm{Lu}$ minous Blue Variables: Massive Stars in Transition, eds. A. Nota \& H.J.G.L.M. Lamers, ASP Conference series vol. 120, p . 326

Waters, L.B.F.M., Morris, P.W., Voors, R.H.M., Lamers, H.J.G.L.M., Trams, N.R.: 1998a, Astrophys. Sp. Sc. 255, 179

Waters, L.B.F.M., et al.: 1998b, Nature 391, 868

Waters, L.B.F.M., et al.: 1998c, A\&A 331, L61

Wolf, B., Zickgraf, F.-J.: 1986, A\&A 164, 435

\section{Discussion}

R. Schulte-Ladbeck: In $\eta$ Car, as I showed in my talk, there is a different grain population in the UV/optical versus the IR. For the optical polarisation, 
we need small grains. The IR polarisation requires large, elongated grains with ferromagnetic inclusions.

S. Shore: That is very interesting since one does not see these, at least not in AG Car. But they are present at some stage in dust forming novae.

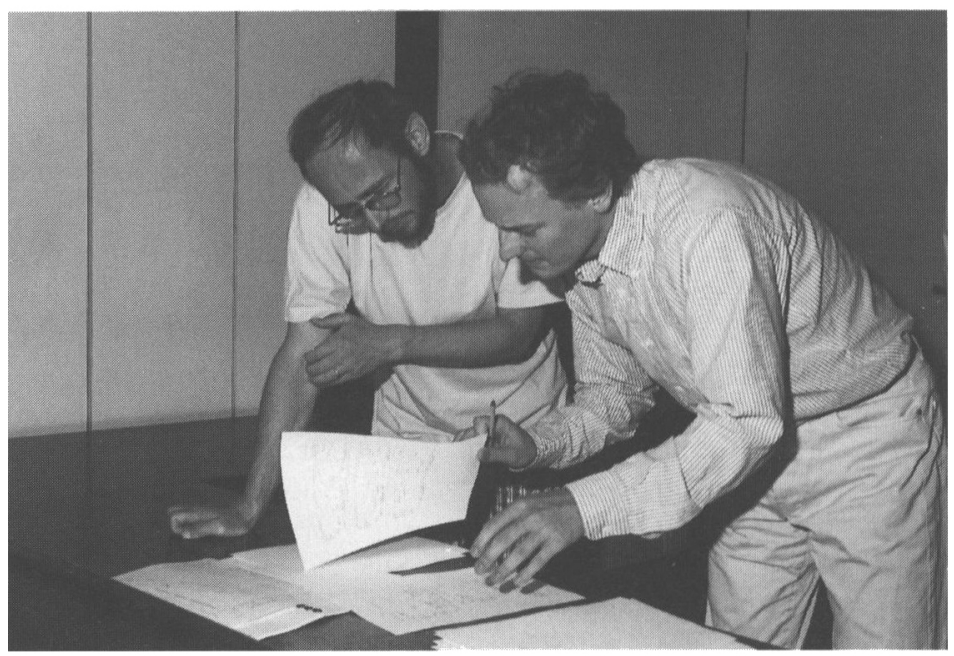

Georges Meynet and Andreas Korn

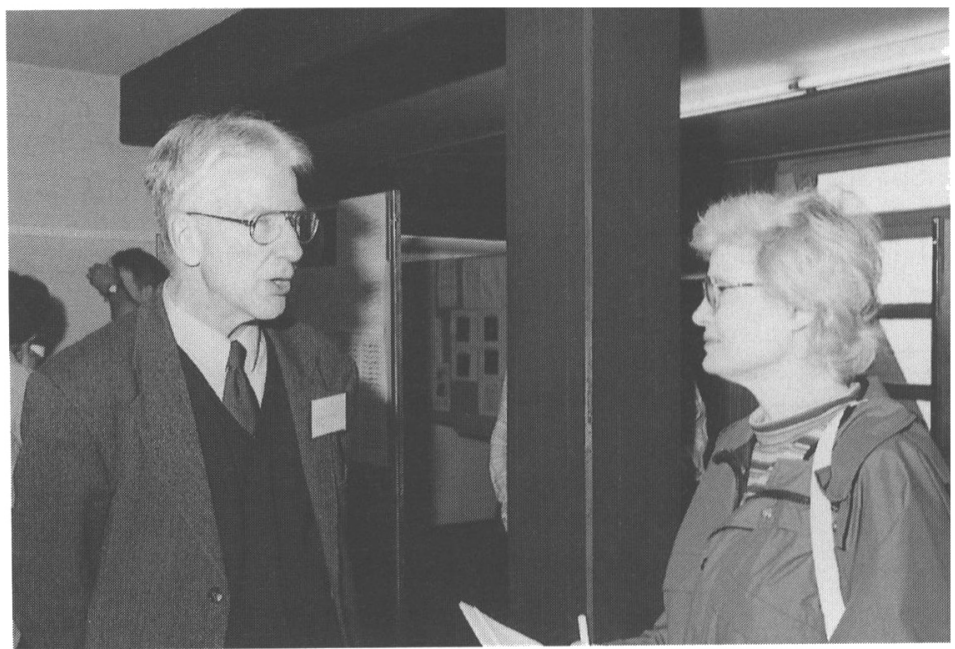

Joachim Dachs and Geraldine Peters 